\title{
Review of Critical Illness Myopathy and Neuropathy
}

\author{
The Neurohospitalist \\ 20I7, Vol. 7(I) 4I-48 \\ (C) The Author(s) 2016 \\ Reprints and permission: \\ sagepub.com/journalsPermissions.nav \\ DOI: $10.1177 / 1941874416663279$ \\ journals.sagepub.com/home/nhos
}

(S)AGE

\author{
Starane Shepherd, MD', Ayush Batra, MD', and David P. Lerner, MD'
}

\begin{abstract}
Critical illness myopathy (CIM) and neuropathy are underdiagnosed conditions within the intensive care setting and contribute to prolonged mechanical ventilation and ventilator wean failure and ultimately lead to significant morbidity and mortality. These conditions are often further subdivided into CIM, critical illness polyneuropathy (CIP), or the combination-critical illness polyneuromyopathy (CIPNM). In this review, we discuss the epidemiology and pathophysiology of CIM, CIP, and CIPNM, along with diagnostic considerations such as detailed clinical examination, electrophysiological studies, and histopathological review of muscle biopsy specimens. We also review current available treatments and prognosis. Increased awareness and early recognition of CIM, CIP, and CIPNM in the intensive care unit setting may lead to earlier treatments and rehabilitation, improving patient outcomes.
\end{abstract}

\section{Keywords}

neuromuscular disease, neurocritical care, muscular disease, polyneuropathies, EMG/NCV

\section{Introduction}

Critical illness myopathy (CIM), critical illness polyneuropathy (CIP), and the overlap, critical illness polyneuromyopathy (CIPNM), are the most common cause of neuromuscular weakness in the intensive care setting and a common cause of failure to wean from the ventilator. Bolton et al first described a small case series of patients with diverse causes for intensive care admission and subsequently for invasive ventilation, of which all of these patients progressed to flaccid quadriparesis, inability to wean from the ventilator and electrodiagnostic findings consistent with a severe motor and sensory polyneuropathy. ${ }^{1}$ Although isolated CIP remains a controversial entity that is likely rare, the incidence of CIM and CIPNM is reported from $25 \%$ to $83 \%$ depending on the underlying critical illness. ${ }^{2,3}$

This diagnosis is typically entertained only when patients fail to wean from ventilatory support, and the true time of onset is often uncertain given concurrent encephalopathy, sedation, and possible paralysis. Up to $62 \%$ of patients who experience failure to wean from the ventilator have some form of neuromuscular weakness. ${ }^{4}$ Common findings in neuromuscular ventilatory failure include decreased maximal inspiratory pressure (also known as negative inspiratory force) greater than $-30 \mathrm{~cm} \mathrm{H}_{2} \mathrm{O}$, decreased maximal expiratory pressure less than $40 \mathrm{~cm} \mathrm{H}_{2} \mathrm{O}$, and decreased forced vital capacity less than $20 \mathrm{~mL} / \mathrm{kg}$. However, patients with respiratory failure for reasons other than neuromuscular failure may have the same abnormal values. In particular, Khalil et al did not find a difference in the maximal inspiratory pressure in those with neuromuscular failure and those with other underlying etiology for inability to wean from the ventilator. ${ }^{5}$ Critical illness myopathy, CIP, and CIPNM not only affect respiration but also most other skeletal muscles.

Presentation varies slightly depending on the underlying disease process. Critical illness myopathy is characterized by more proximal than distal weakness, sensory preservation, and atrophy depending on the duration of illness. Critical illness polyneuromyopathy, on the other hand, is characterized by more distal than proximal weakness, sensory changes, and limited atrophy. Critical illness polyneuromyopathy in turn is characterized by a combination of proximal greater than distal weakness, distal sensory loss, and variable atrophy. Initial preservation of reflexes is common, but gradual loss will occur as weakness progresses in all types. Limitations in the clinical examination of patients in the intensive care unit (ICU) such as the use of sedating

\footnotetext{
' Department of Neurology, Brigham and Women's Hospital \& Massachusetts General Hospital, Harvard Medical School, Boston, MA, USA

Corresponding Author:

David P. Lerner, Department of Neurology, Brigham and Women's Hospital \& Massachusetts General Hospital, Harvard Medical School, 55 Fruit Street, WACC 720, Boston, MA 021 I4, USA.

Email: dplerner@mgh.harvard.edu
} 
medications and intubation status may interfere with reliability. Importantly, bulbar musculature is rarely involved and patients may present with a profound quadriparesis with preservation of facial expression. ${ }^{1,2}$

\section{Diagnosis}

The diagnosis of CIM, CIP, and CIPNM is made via electrodiagnostic studies. Although the differential diagnosis for weakness and ventilatory failure is broad, diffuse weakness of moderate severity warrants evaluation. In conjunction with evaluation for neuromuscular causes of weakness, other etiologies should also be explored. Nonneuromuscular cases for ventilator failure include primary pulmonary, cardiovascular, endocrinologic, and central nervous system diseases. ${ }^{6}$ Other neuromuscular causes within the neural axis can present similarly including Guillain-Barre syndrome, Lambert-Eaton myasthenic syndrome, botulinum toxin exposure, and ongoing neuromuscular blockade that can be evaluated with train-offour testing (Table 1). History and clinical examination may be able to differentiate between these disease entities as CIM, CIP, and CIPNM typically spare the bulbar muscles, lack autonomic instability, and do not result in pupillary changes. Proposed diagnostic criteria for both CIM and CIP are included in Table $2 .^{7-9}$

Although part of the diagnostic criteria for both CIM and CIP, there are many technical challenges to completing nerve conduction studies and electromyography (EMG) in the ICU. Other electronic devices may result in 60-cycle artifact and obscure both nerve conduction and EMG results, thus providing technically inadequate studies for diagnosis. Despite ensuring unnecessary devices are removed from the testing environment, preservation of the technical standards remains challenging. Commonly encountered physiologic changes in the ICU including anasarca and hypothermia also introduce artifact by altering amplitude and velocity recordings for nerve conduction studies. Finally, the clinical status of the patient results in an inability to cooperate with EMG testing causes difficulties interpreting recruitment and interference patterns.

In CIM, the most common electrodiagnostic finding is reduced compound muscle action potential amplitudes, typically below $50 \%$ of lower limit of normal. ${ }^{10}$ Some of these patients have increased action potential duration. These findings can be within 72 hours of admission, and on serial examination, these findings can worsen. ${ }^{11}$ Sensory nerve action potentials are usually normal in CIM, and abnormal results may be due to coexistent neuropathy or common confounders in the ICU like temperature, edema, and recording site. ${ }^{10}$ Decreased muscle membrane excitability is also typically seen in CIM. ${ }^{12}$ Briefly, Rich and Pinter measured direct action potential of the tibialis anterior muscle with stimulation of the fibular nerve and direct muscle stimulation to evaluate membrane excitability. In cases of CIM, the ratio of maximal muscle action potential of the nerve stimulation
Table I. Neuromuscular Differential Diagnosis of "Failure to Wean From Ventilator".

\begin{tabular}{ll}
\hline Motor neuron & Amyotrophic lateral sclerosis \\
Poliomyelitis & Guillain-Barre syndrome \\
& Critical illness polyneuropathy \\
Critical illness polyneuropathy/myopathy & Heavy metal toxicity \\
Vasculitis & Sarcoidosis \\
& Mononeuritis multiplex \\
Neuromuscular junction & Myasthenia gravis \\
& Neuromuscular blockade \\
& Lambert-Eaton myasthenic syndrome \\
& Botulinum toxicity \\
& Organophosphate toxicity \\
& Tetrodotoxin toxicity \\
& Rhabdomyolysis \\
& Mitochondrial myopathy \\
& Muscular dystrophy (eg, Myotonic \\
dystrophy) \\
Critical illness myopathy \\
Acid maltase deficiency
\end{tabular}

to direct muscle stimulation was 1 , and in CIP, the ratio was $<0.5{ }^{12}$ These results have been reproduced using similar but different techniques. ${ }^{13}$ The slowing of direct muscle action potential is correlated with more severe CIM. ${ }^{14}$ Standard needle electrode examination reveals positive waves, fibrillations potentials, or both in $71 \%$ to $100 \% .{ }^{10}$ The motor units are short with low amplitude and polyphasic.

In CIP, the findings resemble a length-dependent axonal polyneuropathy with loss of amplitudes of both sensory nerve action potentials and compound muscle action potentials without significant slowing. ${ }^{10}$ These changes are worse in the legs than the arms and are typically bilateral, although they need not necessarily be symmetric. ${ }^{10}$ The initial findings of CIP occur by week 2 of critical illness but have been reported as early as 72 hours from admission to the ICU. ${ }^{15}$ Standard needle examination reveals fibrillation potentials and positive waves in distal and proximal muscles, which typically occur within 2 weeks of illness but can also be seen as early as 1 week. ${ }^{10,14,16}$ Motor unit potentials demonstrate decreased recruitment and morphology changes consistent with the timing of the study. ${ }^{14}$ Interestingly, fibrillation potentials can also be demonstrated within facial musculature. ${ }^{10}$

Respiratory failure and inability to wean from ventilator support are common symptoms of the critical illness-acquired weaknesses, and examination of the phrenic nerve and diaphragm can assist with diagnosis. Diaphragm EMG can show fibrillations and positive sharp waves or reduced number of motor unit potentials. ${ }^{17}$ Phrenic nerve conduction can demonstrate either prolonged latencies or reduced motor unit action potentials. ${ }^{17}$ Limited diaphragmatic excursion during spontaneous breathing trials is consistent with diaphragmatic weakness but is not limited to weakness acquired in the ICU. ${ }^{18}$ 
Table 2. Suggested Diagnostic Criteria for Critical IIIness Polyneuropathy and Critical IIlness Myopathy. ${ }^{\text {a }}$

\begin{tabular}{|c|c|}
\hline CIP & CIM \\
\hline Critically ill (sepsis and multi-organ failure) & $\begin{array}{l}\text { Not required; typically exposed to variable combination of } \\
\text { neuromuscular blocking agent and corticosteroids in the setting of } \\
\text { sepsis and multi-organ failure }\end{array}$ \\
\hline Limb weakness is present & Limb weakness is present \\
\hline $\begin{array}{l}\text { Difficulty in weaning from mechanical ventilatory support with the } \\
\text { exclusion of cardiac and pulmonary causes }\end{array}$ & $\begin{array}{l}\text { Difficulty in weaning from mechanical ventilatory support with the } \\
\text { exclusion of cardiac and pulmonary causes }\end{array}$ \\
\hline & $\begin{array}{l}\text { 3. Normal repetitive nerve simulation, and EMG with short- } \\
\text { duration, low-amplitude motor unit potential with early full or } \\
\text { normal recruitment of motor unit action potentials }\end{array}$ \\
\hline
\end{tabular}

Abbreviations: CIM, critical illness myopathy; CIP, critical illness polyneuromyopathy; EMG, electromyography.

${ }^{\text {a Adapted from Bolton }}{ }^{7}$ and Lacomis et al. ${ }^{8}$

Although useful for diagnosis, diaphragmatic EMG remains technically challenging within the ICU setting.

With the known limitation of nerve conduction studies within the ICU, muscle biopsy may be useful for definitive diagnosis. Raghig et al describe a case series of 10 patients with a clinical diagnosis of CIM, CIP, or CIPNM, and pathological specimen was concordant with clinical diagnosis in 9 of the 10 patients and 3 cases where biopsy identified an underlying disease when clinical or electrodiagnosis could not. ${ }^{19}$ The typical pathological findings in CIM include relative selective loss of myosin and loss of type 2 greater than type 1 fibers. ${ }^{20}$ Amato and Russell describe and demonstrate common muscle biopsy features. ${ }^{21}$ Muscle biopsy in CIP will demonstrate features of denervation and reinnervation with small muscle fibers, fiber-type grouping, and fiber group atrophy. ${ }^{9}$ Nerve biopsy in CIP will demonstrate widespread axonal degeneration of both motor and sensory nerves. ${ }^{9}$ Muscle biopsy in CIPNM can have features of both CIM, typically thick filament loss, and CIP, and denervation and reinnervation changes and nerve biopsy can have axonal degeneration. ${ }^{9}$

\section{Epidemiology}

The incidence of neuromuscular weakness in the ICU is specific to the underlying disease process, diagnostic criteria used, and timing of diagnostic evaluation. ${ }^{22}$ The initial case reports demonstrated similar findings in patients with varying underlying pathologies - trauma, sepsis, respiratory failure. ${ }^{1}$ In patients with status asthmaticus who require intubation and treatment with intravenous steroids, at least $33 \%$ will develop CIM. $^{23}$ In sepsis and systemic inflammatory response syndrome, the rate is much higher at $70 \%$, and those with further complications of multiorgan dysfunction have reported rate of
$100 \%{ }^{24}$ Acute respiratory distress syndrome can also result in CIM/CIP with reported rates of $60 \% .^{25}$ In all patients who receive mechanical ventilation for at least 4 to 7 days, the occurrence of CIM/CIP was $25 \%$ to $33 \%$ on clinical examination and up to $58 \%$ to $68 \%$ on electrodiagnostic testing. ${ }^{3,14}$ In pooled analyses of reports, Stevens et al report an overall rate of $46 \%$ diagnosis of CIPNM. ${ }^{22}$

There are specific disease processes that increase the risk of CIM/CIP/CIPNM, and other disease variabilities can potentially increase the risk. Patients with CIPNM have higher blood glucose, and in a randomized trial of conventional (blood glucose target 180-200 mg/dL) and intensive (blood glucose target $80-110 \mathrm{mg} / \mathrm{dL}$ ) blood glucose control, the conventional blood glucose had higher rates of CIM/CIP/ CIPNM - odds ratio (OR) of 2.6 (95\% confidence interval [CI]: 1.6-4.2). ${ }^{22,26}$ There are conflicting data on the risk of renal replacement therapy and odds of developing CIM/CIP/ CIPNM. $^{3,26}$ Female gender is an independent risk factor for the development of CIPNM. ${ }^{3}$ Higher underlying disease burden, as demonstrated by higher Acute Physiologic and Chronic Health Evaluation III score and Sequential Organ Failure Assessment score, also have increased the risk of CIPNM. $^{27,28}$ Mechanical ventilation has been studied extensively in relation to CIPNM. All studies except one demonstrate increased duration of mechanical ventilation in patients with CIPNM, and CIPNM has been shown to predict duration of mechanical ventilation and failure to wean. ${ }^{22,29-31}$

Because initial descriptions of CIM were in those receiving high-dose steroids and neuromuscular blockade, linkage of the disease to these medications was inferred. There are multiple studies examining the relationship between medications and CIM/CIP/CIPNM. Most of these do not demonstrate the increased risk of CIPNM with the use of glucocorticoids or 


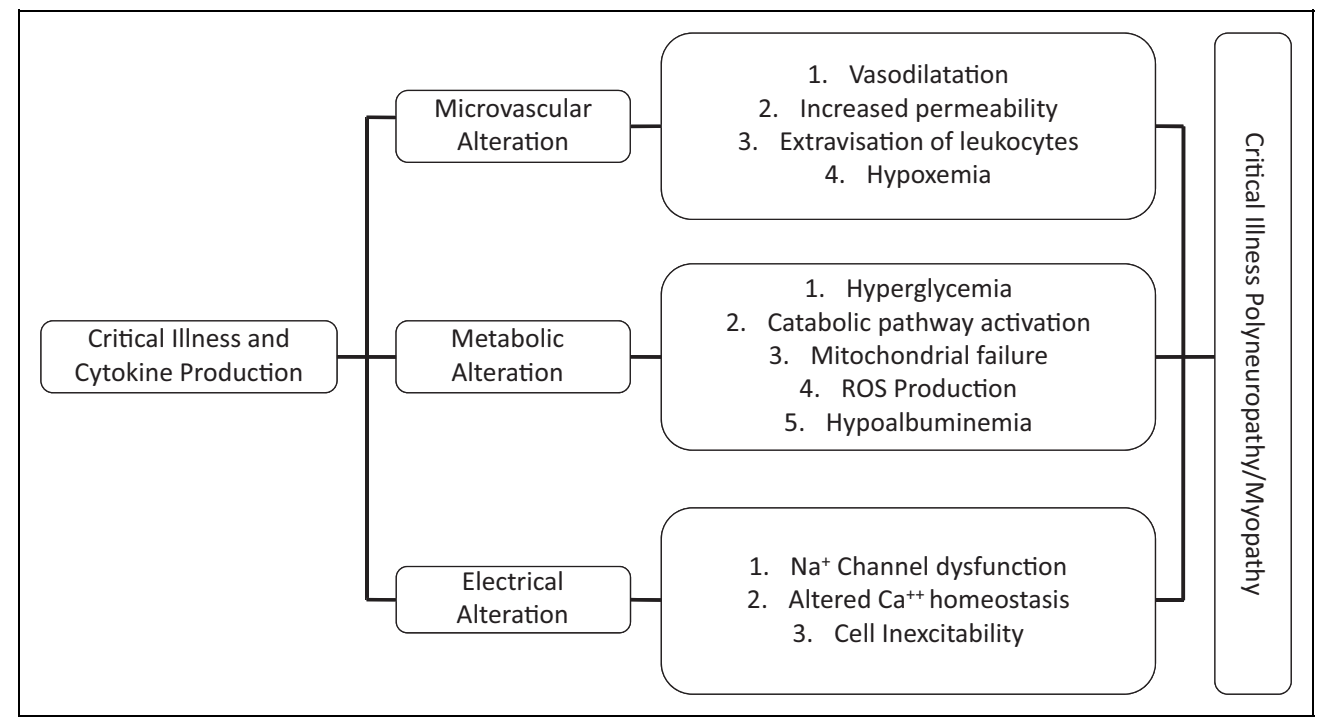

Figure I. Pathophysiology of critical illness polyneuropathy and myopathy.

neuromuscular blockade. ${ }^{22}$ Results remain varied, however, in 1 of the 2 studies evaluating CIPNM, multivariate analysis of CIPNM and the use of glucocorticoids demonstrated increased OR (14.9 [95\% CI: 3.2-69.8]). ${ }^{3}$ Garnacho-Montero et al demonstrated an increased OR between CIPNM and neuromuscular blockers (OR: 16.32 [95\% CI: 1.34-199]). ${ }^{31}$ Three individual reports have demonstrated the association between vasopressor drug administration and the likelihood of developing CIPNM. ${ }^{27,32,33}$ However, these results may not be widely applicable to the general ICU population given that they are specific to cardiothoracic ICU admissions for cardiac surgery.

\section{Pathophysiology}

Although CIM, CIP, and CIPNM are present similarly clinically within the same patient population, there are drastic differences in the underlying pathology. Interestingly, the electrophysiological changes found during testing can occur within hours of admission to the ICU. ${ }^{34}$ Figure 1 represents a simplified view of the underlying pathophysiology of CIM, CIP, and CIPNM. ${ }^{35,36}$ Muscles and nerves are not isolated from the microcirculatory, cellular, and metabolic derangements that occur during critical illness.

Critical illness myopathy is a heterogenous entity with differing pathologic subtypes - traditional CIM demonstrates myosin loss, fulminant acute necrotizing myopathy, and cachectic myopathy. ${ }^{37-39}$ Acute necrotizing myopathy commonly has marked elevation in creatinine kinase and myoglobinuria. ${ }^{37}$ Cachectic myopathy appears similar to disuse atrophy with marked wasting and biopsy consistent with type 2 fiber loss. ${ }^{39}$ Although each subtype results in the same clinical phenotype, there is likely differing underlying pathophysiologic processes that have yet to be fully elucidated.

The more traditional CIM is defined by loss of the myosin and further breakdown of the contractile apparatus. ${ }^{37-40}$ This
CIM will typically have normal to slightly elevated creatinine kinase values. ${ }^{39}$ Experimental models have demonstrated denervated muscles exposed to high-dose corticosteroids will have selective thick filament loss, and the number of corticosteroid receptors is increased following denervation. ${ }^{39}$ The process by which steroids result in these changes is not fully understood, and other toxins or physiologic stressors may be the cause of myosin loss including increased catabolic demands resulting in generalized muscle wasting. ${ }^{41}$

There has also been demonstration of muscle fiber membrane dysfunction in CIM. Allen et al studied direct muscle action potentials with needle electrodes within the tibialis anterior muscle demonstrating normal nerve conduction velocities, but within a single muscle fiber, slowing of the conduction suggesting that muscle fiber membranes are unable to transduce signals. ${ }^{15}$ It is uncertain if the abnormal sarcolemma conductance is the result of or cause of the resultant myosin loss. ${ }^{39}$ This can, in part, be caused by the inactivation of sodium channels as demonstrated in animal models. ${ }^{12,42}$

The exact mechanism of axonal injury in CIP is unknown. One proposed mechanism of injury is microcirculatory changes associated with systemic inflammation. ${ }^{20}$ Increased production of cytokines, nitric oxide, and other reactive oxygen species results in a change to anaerobic oxidation, ${ }^{20,22}$ which leads to changes in distal nerve ischemia and degeneration. ${ }^{7}$

A second proposed mechanism of neuronal dysfunction in CIP is related to increased vascular permeability with increased leukocytes within the endoneurial space and formation of edema. ${ }^{43}$ As with the central nervous system, hypoalbuminemia and hyperglycemia can worsen edema. ${ }^{44}$ Additionally, sodium channel inactivation resulting in rapid hypoexcitability of peripheral nerves has been implicated in the pathophysiology of nerve dysfunction. ${ }^{42}$

As mentioned in both CIP and CIM, there is inactivation of sodium channels and change in resting membrane 
potential. ${ }^{12,42}$ Coexisting CIP can potentiate the CIM that underscores the importance of potential denervation within the pathogenesis of CIM. ${ }^{39}$ This supports the hypothesis that CIM and CIP are different clinical manifestations of the same underlying disease process. ${ }^{45}$

\section{Treatment/Prognosis}

Patients with neuromuscular weakness have increased length of stay and more ventilator-dependent days than those without. ${ }^{9,46}$ Nine of the 12 studies reported no difference in ICU mortality between patients with and without CIPNM, but hospital mortality was higher in 3 of the 7 reports. ${ }^{47,48}$ Mortality rates vary from $16 \%$ to $55 \%$, and of those patients who do survive, most face significant chronic long-term complications. $^{24,49}$

The chronic sequela following admission to the ICU is multifactorial, including psychological stress, pulmonary insufficiency, pain, and motor weakness as their primary functional impairment. ${ }^{50}$ Twenty percent of patients diagnosed with CIM, CIP, or CIPNM have ongoing weakness or sensory changes at the time of discharge ${ }^{50}$ However, there are divergent recovery paths among the different diseases. Guarneri et al followed patients with electrodiagnostic diagnosed CIM, CIP, or CIPNM and found that 11 of the 12 patients with CIM had complete recovery by 6 months, but those with CIP and CIPNM had lower rates of recovery and less overall improvement -2 of the 7 with recovery. ${ }^{50}$ The consistent theme that CIM recovers more quickly and more completely than CIP or CIPNM has been demonstrated many times. ${ }^{3,50-52}$ On follow-up electrodiagnostic evaluation, those with isolated CIM do not have ongoing myopathic changes at 12 months or greater ${ }^{51}$ On repeat electrodiagnosis for patients with evidence of neuropathy testing greater than 1 year from discharge, over $80 \%$ of patients have evidence of polyneuropathy. ${ }^{51}$ Ongoing severe disability is reported between $14.2 \%$ and $66.7 \% .^{50-53}$ Those that will recover, the improvement is typically seen within the first 6 to 12 months. ${ }^{3,54-56}$ Recovery can continue for up to 24 months, as Intiso et al reported of those that have complete recovery, $23 \%$ occurred between 12 and 24 months. ${ }^{54}$

The major risk factors for development of CIM, CIP, or CIPNM are systemic inflammatory response syndrome; sepsis; hyperglycemia; female sex; vasopressor administration; and mixed data on renal replacement therapy, steroids, and neuromuscular blockade. 1,3,22,26,27,31 The treatment of sepsis is discussed in length elsewhere and beyond the scope of this discussion. ${ }^{56}$ Of particular interest to treatment and prevention of CIM, CIP, and CIPNM is hyperglycemia, as tight glucose control remains the only intervention that has demonstrated a clinical benefit. Conventional glucose control versus intensive glucose control has an increased OR of 2.6 (95\% CI: 1.6-4.2) of CIPNM diagnosis made by electrodiagnostic studies $(52 \%$ vs $29 \%) .{ }^{54,57}$ Follow-up studies have demonstrated increased mortality in patients with strict normoglycemia protocols, and the appropriate management strategy remains debated. ${ }^{58}$ As discussed in the pathophysiology section, there is an inflammatory response that may contribute to CIM, CIP, CIPNM, and treatment with immunomodulation has also been tried. Mohr et al's initial retrospective review found potentially improved outcomes with intravenous immunoglobulin (IVIG) treatment; however, a randomized control trial of IVIG in electrodiagnostic confirmed CIM, CIP, and CIPNM did not demonstrate any benefit and was terminated early. ${ }^{29,59}$ Research continues to demonstrate modulation of proinflammatory pathways may be beneficial; one of which, growth differentiation factor-15, may undergo further evaluation for the treatment of ICU-acquired weakness. ${ }^{60}$

Although there are no randomized control trials published evaluating physical rehabilitation interventions in CIM, CIP, or CIPNM, early ICU mobility can decrease length of stay. ${ }^{61,62}$ Limited case series exist that evaluate physical therapy within the ICU on patients with ICU-acquired weakness. Ali et al describe 35 patients with intermittent physical therapy versus 19 patients who received 30 minutes a day for 5 days a week of intensive physical therapy. Of those with increased physical therapy time, $32 \%$ were ultimately discharged to home versus $11 \%$ in the intermittent group, whereas both groups had $57 \%$ of patients discharged to rehabilitation. ${ }^{63}$ Nordon-Craft et al completed a similar 30 minutes a day for 5 days-a-week physical therapy evaluation on patients intubated for greater than 7 days and demonstrated safety and feasibility of the intervention. ${ }^{64}$ Interestingly, other studies have looked at evaluating all patients in the ICU, and although early mobility and physical therapy in the ICU may improve short- and long-term functional outcome, there does not appear to be a change in the rate of ICU-acquired weakness. ${ }^{62,65}$ For patients who are unable to participate in physical therapy, evaluation of passive mechanical loading has demonstrated improved force generation but was limited by not characterizing underlying electrodiagnostic abnormalities. ${ }^{66}$ Finally, direct muscle electrical stimulation within the ICU has shown less decrease cross-sectional area of muscle mass with stimulation for 30 minutes daily. ${ }^{67}$ This was followed by a larger study of 52 patients who were randomized to 55 minutes daily of bilateral lower extremity electrical stimulation and demonstrated decreased rate of examination-defined CIPNM (12.5\% vs 39.3\%; OR: 0.22 [CI:0.05-0.92; $P=.04]$ ), decreased weaning time (median 1 vs 3 days; $P=.003$ ), and improved motor examination scores (median 55 vs $52 ; P=.04) .{ }^{68}$

Although there are limited treatments directed specifically at ICU-acquired weakness, it is important to anticipate and prevent complications of profound weakness. As with all immobilized patients, appropriate deep vein thrombosis prophylaxis is paramount. Although there are limitations on when prophylactic treatment can start, earlier initiation is preferred. Appropriate positioning of limbs to prevent pressure ulcer development as well as focal compression palsies is the critical preventative measure in this patient population. 
Additionally, patients should undergo regular full skin evaluations for early ulcer development detection and treatment. Although early nutrition initiation may not change the amount of muscle wasting that occurs during ICU admission, it has been shown to improve ICU outcomes. ${ }^{69}$

\section{Conclusion}

Given the prevalence of weakness in the ICU, recognizing neuromuscular etiologies of weakness is imperative to ensuring the best potential functional outcome for these patients. Thorough evaluation of causes for potential neuromuscular weakness is warranted to determine the appropriate treatment, and identification of systemic or medical causes of weakness has profoundly different treatment approaches than those secondary to CIM or neuropathy. Although clinical examination for neuromuscular weakness can be challenging in the ICU setting, the use of electrodiagnosis and in the appropriate clinical context, muscle biopsy, can help diagnose CIM, CIP, or CIPNM. These conditions may have overlapping clinical presentations, most commonly first recognized when patients fail to wean successfully from ventilator support. However, typical patterns of weakness can potentially be elicited at the bedside and may help distinguish between CIM and CIP in some cases. Although the prevalence of CIM, CIP, and CIPNM within the ICU depends in part on the underlying condition leading to intensive care admission, numerous studies have demonstrated that systemic inflammatory processes infer a higher risk of development. ${ }^{14,35,41}$ Pathophysiologic differences in the underlying myopathy, neuropathy, or polyneuromyopathy seem to account for the variable prognosis and rate of recovery for patients.

Proposed treatments for CIM, CIP, and CIPNM initially focused on modulating the suspected inflammatory triggers, however, no randomized controlled trial has shown benefit for either IVIG or plasmapheresis for improved recovery in this population. More targeted approaches at selected inflammatory cascade pathways may provide a more suitable pharmacologic treatment approach in the near future. Nevertheless, early mobilization and early initiation of physical therapy consistently portends a better functional outcome for patients with confirmed CIM, CIP, or CIPNM in numerous limited studies. This remains a feasible and promising area for continued research to improve treatment options, and perhaps, system-based approaches to ICU bedside care may allow for further gains to be made with these treatment strategies. Ultimately, recognizing these conditions early and subsequently tailoring care remain the most important treatment aspect for CIM, CIP, and CIPNM.

\section{Authors' Note}

Starane Shepherd and Ayush Batra contributed equally to the drafting and revision of this manuscript.

\section{Declaration of Conflicting Interests}

The authors declared no potential conflicts of interest with respect to the research, authorship, and/or publication of this article.

\section{Funding}

The authors received no financial support for the research, authorship, and/or publication of this article.

\section{References}

1. Bolton CF, Gilbert JJ, Hahn AF, Sibbald WJ. Polyneuropathy in critically ill patients. J Neurol Neurosurg Psychiatry. 1984; 47(11):1223-1231.

2. Linos K, Foot C, Ziegenfuss M, Freeman WD, Tan KM. Critical illness weakness: common questions. Curr Anesth Crit Care. 2007; 18:252-260.

3. De Jonghe B, Sharshar T, Lefaucheur JP, et al. Paresis acquired in the intensive care unit: a prospective multi-center study. JAMA. 2002;288(22):2859-2867.

4. National Heart, Lung and Blood Institute (NHLBI) workshop on respiratory muscle fatigue: a report of the respiratory muscle fatigue workshop group. Am Rev Resp Dis. 1990;142(2): 474-480.

5. Khalil Y, El Din Mustafa E, Youssef A, Imam HM, Behairy AE. Neuromuscular dysfunction associated with delayed weaning from mechanical ventilation in patients with respiratory failure. Alexandria J Med. 2012;48(3):223-232.

6. Heunks LE, van der Hoeven JG. Critical review: the ABC of weaning failure - a structured approach. Crit Care. 2010; 14(6):245.

7. Bolton CF. Neuromuscular manifestation of critical illness. Muscle Nerve. 2005;32(2):140-163.

8. Lacomis D, Zochodne DW, Bird SJ. Critical illness myopathy: what's in a name? Muscle Nerve. 2000;23(12):1767-1772.

9. Visser LH. Critical illness polyneuropathy and myopathy clinical features, risk factors and prognosis. Eur J Neurol. 2006; 13(11):1203-1212.

10. Lacomis D. Electrophysiology of neuromuscular disorders in critical illness. Muscle Nerve. 2013;47(3):452-463.

11. Bednarik J, Lukas Z, Vondracek. Critical illness polyneuromyopathy: the electrophysiological components of a complex entity. Intensive Care Med. 2003;29(9):1505-1514.

12. Rich M, Pinter MJ. Crucial role of sodium channel fast inactivation in muscle fiber inexcitability in a rat model of critical illness myopathy. J Physiol. 2003;547(pt 2):555-566.

13. Trojaborg W, Weimer LH, Hays AP. Electrophysiologic studies in critical illness associated weakness: myopathy or neuropathy a reappraisal. Clin Neurophyiol. 2001;112(9):1586-1593.

14. Leijten FS, De Weerd AW, Poortvliet DC, De Ridder VA, Ulrich C, Harink-De Weerd JE. Critical illness polyneuropathy in multiple organ dysfunction syndrome and weaning from the ventilator. Intensive Care Med. 1996;22(9):856-861.

15. Allen DC, Arunachalam R, Mills KR. Critical illness myopathy: further evidence from muscle-fiber excitability studies of an acquired channelopathy. Muscle Nerve. 2008;37(1):14-22. 
16. Tennila A, Salmi T, Pettila V, Roine RO, Varpula T, Takkunen O. Early signs of critical illness polyneuropathy in the ICU patients with systemic inflammatory response syndrome or sepsis. Intensive Care Med. 2000;26(9):1360-1363.

17. Zifko UA, Zipko HT, Bolton CF. Clinical and electrophysiological findings in critical illness polyneuropathy. J Neurol Sci. 1998;159(2):186-193.

18. Madams D, Soilemezmi E, Tsagourias M, et al. Sonographic evaluation of the diaphragm in critically ill patients. Technique and clinical applications. Intensive Care Med. 2013;39(5): 801-810.

19. Raghig H, Young GB, Hammond R, Nicolle M. A comparison of EMG and muscle biopsy in ICU weakness. Neurocrit Care. 2010;13(3):326-330.

20. Di Giovanni S, Mirabella M, D’Amico A, Tonali P, Servidei S. Apoptotic feature accompany acute quadriplegic myopathy. Neurology. 2000;55(6):854-858.

21. Amato AA, Russell JA. Myopathies associated with system disease. In: Neuromuscular Disorders. New York, NY: McGrawHill Companies; 2008:721-736.

22. Stevens RD, Dowdy DW, Michaels RK, Mendez-Tellez PA, Pronovost PJ, Needham DM. Neuromuscular dysfunction acquired in critical illness: a systematic review. Intensive Care Med. 2007;33(11):1876-1891.

23. Douglass JA, Tuxen D, Horne M, et al. Myopathy in severe asthma. Am Rev Respir Dis. 1992;146(2):517-519.

24. Witt NJ, Zochodne DW, Bolton CF, et al. Peripheral nerve function in sepsis and multiple organ failure. Chest. 1991;99(1): 176-184.

25. Bercker S, Weber-Carsten S, Deja M, et al. Critical illness polyneuropathy and myopathy in patients with acute respiratory distress syndrome. Crit Care Med. 2005;33(4):711-715.

26. Van den Berghe G, Wilmer A, Hermans G, et al. Intensive insulin therapy in the critically ill patients. $N$ Engl J Med. 2001; 354(19):1359-1367.

27. Habib FA. Respiratory failure after surgery or trauma. In: Stephen M. Cohn, ed. Complications in Surgery and Trauma. Boca Raton, FL: Taylor \& Francis Group; 2006:271-86.

28. Beknarik J, Vondracek P, Dusek L, Moravcova E, Cundrie I. Risk factors for critical illness polyneuromyopathy. J Neurol. 2005;252(3):343-351.

29. Mohr M, Englisch L, Roth A, Burchardi H, Zielmann S. Effects of early treatment with immunoglobulin on critical illness polyneuropathy following multiple organ failure and gram-negative sepsis. Intensive Care Med. 1997;23(11):1144-1149.

30. De Jonghe B, Bastuji-Garin S, Sharshar T, Putin H, Brouchard L. Dose ICU-acquired paresis lengthen weaning from mechanical ventilation? Intensive Care Med. 2004;30(6):1117-1121.

31. Garnacho-Motero J, Amaya-Villar R, Garcia-Garmendia JL, Madrazo-Osuna J, Ortiz-Leyba C. Effect of critical illness polyneuropathy on the withdrawal from mechanical ventilation and the length of stay in septic patients. Crit Care Med. 2005;33(2): 349-354.

32. Thiele RI, Jakob H, Hund E, et al. Sepsis and catecholamine support are the major risk factors for critical illness polyneuropathy after open heart surgery. Thorac Cardiovasc Surg. 2000;48(3):145-150.

33. Thiele RI, Jakob H, Hund E, et al. Critical illness polyneuropathy: a new iatrogenically induced syndrome after cardiac surgery? Eur J Cardiothorac Surg. 1997;12(6):826-835.

34. Fink MP, Evans TW. Mechanisms of organ dysfunction in critical illness: report from a round table conference held in Brussels. Intensive Care Med. 2002;28(3):369-375.

35. Hermans G, De Jonghe B, Bruyninckx F, Van den Berghe G. Clinical review: critical illness polyneuropathy and myopathy. Crit Care. 2008;12(6):238-244.

36. Zhou C, Wu L, Fenghimg Ni, Ji W, Wu J, Zhang H. Critical illness polyneuropathy and myopathy: a systematic review. Neural Regen Res. 2014;9(1):101-110.

37. Waclawik AJ, Sufit RL, Beinlich BR, Schutta HS. Acute myopathy with selective degeneration of myosin filaments following status asthmaticus treated with methylprednisolone and vecuronium. Neuromuscul Disord. 1992;2(1):19-26.

38. Larsson L, Li X, Edstrom L, et al. Acute quadriplegia and loss of muscle myosin in patients treated with non-depolarizing neuromuscular blocking agents and corticosteroids: mechanisms at the cellular and molecular levels. Crit Care Med. 2000;28(1):34-45.

39. Hund E. Neurological complications of sepsis: critical illness polyneuropathy and myopathy. J Neurol. 2001;248(11):929-934.

40. Ramsay DA, Zochodne DW, Robertson DM, Nag S, Ludwin SK. A syndrome of acute severe muscle necrosis in intensive care unit patients. J Neuropathol Exp Neurol. 1993;52(4):387-398.

41. Latronico N, Bolton CF. Critical illness polyneuropathy and myopathy: a major cause of muscle weakness and paralysis. Lancet Neurol. 2011;10(10):931-941.

42. Novak KR, Nardelli P, Cope TC, et al. Inactivation of sodium channel underlies reversible neuropathy during critical illness in rats. J Clin Invest. 2009;199(5):1150-1158.

43. Fenzi F, Latronico N, Refatti N, Rizzuto N. Enhanced expression of E-selectin on the vascular endothelium of peripheral nerve in critically ill patients with neuromuscular disease. Acta Neuropathol. 2003;160(1):75-82.

44. Latronisco N, Peli E, Botteri M. Critical illness myopathy and neuropathy. Curr Opin Crit Care. 2005;11(2):126-132.

45. Khan J, Harrison TB, Rich MM. Mechanisms of neuromuscular dysfunction in critical illness. Crit Care Clin. 2008;24(1):165-172.

46. Barlas I, Oropello JM, Benjamin E. Neurologic complications in the intensive care. Curr Opin Crit Care. 2001;7(2):68-73.

47. Amaya-Villar R, Garnacho-Montero J, Carcia-Garmendia JL, et al. Steroid-induced myopathy in patients intubated due to exacerbation of chronic obstructive pulmonary disease. Intensive Care Med. 2005;31(1):157-161.

48. Campellone JV, Lacomis D, Kramer DJ, Van Cott AC, Giuliani MJ. Acute myopathy after liver transplantation. Neurology. 1998;50(1):46-53.

49. Leitjen FS, Harnick-de Weerd JE, Poorvliet DC, de Weerd AW. The role of polyneuropathy in motor convalescence after prolonged mechanical ventilation. JAMA. 1995;274(15):1221-1225.

50. Guarneri B, Bertolini G, Latronico N. Long-term outcome in patients with critical illness myopathy or neuropathy: the Italian 
multicentre CRIMYNE study. J Neurol Neurosurg Psychiatry. 2008;79(7):838-841.

51. Zifko UA. Long-term outcome of critical illness polyneuropathy. Muscle Nerve Suppl. 2000;9:S49-S52.

52. Lacomis D, Petrella JT, Guiliani MJ. Causes of neuromuscular weakness in the intensive care unit: a study of ninety-two patients. Muscle Nerve. 1998;21(5):610-617.

53. Daffurn K, Bishop GF, Hillman KM, Bauman A. Problems following discharge after intensive care. Intensive Crit Care Nurs. 1994;10(4):244-251.

54. Intiso D, Amoruso L, Zarrelli M, et al. Long-term functional outcome and health status of patients with critical illness polyneuromyopathy. Acta Neorl Scand. 2001;123(3):211-219.

55. Berek K, Margreiter J, Willeit J, Berek A, Schmutzhard E, Mutz NJ. Polyneuropathies in critically ill patients: a prospective evaluation. Intensive Care Med. 1996;22(9):849-855.

56. Dellinger RP, Levy MM, Rhodes A, et al; Surviving Sepsis Campaign Guidelines Committee including The Pediatric Subgroup. Surviving Sepsis Campaign: international guidelines for management of severe sepsis and septic shock. Intensive Care Med. 2013;39(2):165-228.

57. Hermans G, Wilmer A, Meersseman W, et al. Impact of intensive insulin therapy on neuromuscular complications and ventilator dependency in the medical intensive care unit. $\mathrm{Am} J$ Respir Crit Care Med. 2007;175(5):480-489.

58. NICE-SUGAR Study Investigators; Finfer S, Chittock DR, Su $\mathrm{SY}$, et al. Intensive versus conventional glucose control in critically ill patients. N Eng J Med. 2009;360(13):1283-1297.

59. Brunner R, Rinner W, Haberler C, et al. Early treatment with IgM-enriched intravenous immunoglobulin dose not mitigate critical illness polyneuropathy and/or myopathy in patients with multi-organ failure and SIRRS/sepsis: a prospective, randomized, placebo-controlled, double-blinded trial. Crit Care. 2013;17(5):R213.
60. Hermans G, Van der Berghe G. Clinical review: intensive care unit acquired weakness. Crit Care. 2015;19:R274.

61. Mehrholz J, Phol M, Kugler J, Burridge J, Muckel S, Elsner B. Physical rehabilitation for critical illness myopathy and neuropathy. Cochrane Database Syst Rev. 2015;(3):CD010942.

62. Morris PE, Goad A, Thompson C, et al. Early intensive care unit mobility therapy in the treatment of acute respiratory failure. Crit Care Med. 2008;36(8):2238-2243.

63. Ali NA, O'Brien JM Jr, Hoffman SP, et al; Midwest Critical Care Consortium. Acquired weakness, handgrip strength and mortality in critically ill patients. Am J Respir Crit Care Med. 2008;178(3):261-268.

64. Nordon-Craft A, Schenkman M, Ridgeway K, Benson A, Moss M. Physical therapy management and patient outcomes following ICU-acquired weakness: a case series. J Neurol Phys Ther. 2011;35(3):133-140.

65. Schweicket WD, Pholman MC, Pohlman AS, et al. Early physical and occupational therapy in mechanically ventilated, critically ill patients: a randomized control trial. Lancet. 2009; 373(9678):1874-1882.

66. Llano-Diez M, Renaud G, Andersson M, et al. Mechanisms underlying ICU muscle wasting and effects of passive mechanical loading. Crit Care. 2012;16(5):R209.

67. Gerovasili V, Stefanidis K, Vitzilaios K, et al. Electrical muscle stimulation preserves the muscle mass of critically ill patients: a randomized study. Crit Care. 2009;13(5):R161.

68. Routsi C, Gerovasili V, Vasileiadis I, et al. Electrical muscle stimulation prevents critical illness polyneuromyopathy: a randomized parallel intervention trial. Crit Care. 2010; 14(2):R74.

69. Hermans G, Casaer MP, Clerckx B, et al. Effect of tolerating macronutrient deficit on the development of intensive-care unit acquired weakness: a subanalysis of the EPaNIC trial. Lancet Respir Med. 2013;1(8):621-629. 